\title{
The metabolic syndrome - a neuroendocrine disorder?
}

\author{
Per Björntorp* and Roland Rosmond \\ Department of Heart and Lung Diseases, University of Göteborg, Sahlgren's Hospital, S-413 45 Göteborg, Sweden
}

\begin{abstract}
Central obesity is a powerful predictor for disease. By utilizing salivary cortisol measurements throughout the day, it has now been possible to show on a population basis that perceived stressrelated cortisol secretion frequently is elevated in this condition. This is followed by insulin resistance, central accumulation of body fat, dyslipidaemia and hypertension (the metabolic syndrome). Socio-economic and psychosocial handicaps are probably central inducers of hyperactivity of the hypothalamic-pituitary adrenal (HPA) axis. Alcohol, smoking and traits of psychiatric disease are also involved. In a minor part of the population a dysregulated, depressed function of the HPA axis is present, associated with low secretion of sex steroid and growth hormones, and increased activity of the sympathetic nervous system. This condition is followed by consistent abnormalities indicating the metabolic syndrome. Such 'burned-out' function of the HPA axis has previously been seen in subjects exposed to environmental stress of long duration. The feedback control of the HPA axis by central glucocorticoid receptors (GR) seems inefficient, associated with a polymorphism in the $5^{\prime}$ end of the GR gene locus. Homozygotes constitute about $14 \%$ of Swedish men (women to be examined). Such men have a poorly controlled cortisol secretion, abdominal obesity, insulin resistance and hypertension. Furthermore, polymorphisms have been identified in the regulatory domain of the GR gene that are associated with elevated cortisol secretion; polymorphisms in dopamine and leptin receptor genes are associated with sympathetic nervous system activity, with elevated and low blood pressure, respectively. These results suggest a complex neuroendocrine background to the metabolic syndrome, where the kinetics of the regulation of the HPA axis play a central role.
\end{abstract}

Metabolic syndrome: HPA axis: Stress: Glucocorticoid receptor

The metabolic syndrome or syndrome X (Reaven, 1988) is a cluster of symptoms generally considered to include insulin resistance, central obesity, dyslipidaemia and hypertension. Insulin resistance has been considered to be the central feature, because dyslipidaemia and perhaps hypertension may be secondary consequences to the prevailing hyperinsulinaemia (Reaven, 1988). Central obesity is, however, more difficult to place in a pathogenetic context along this line of explanation.

Obesity is now developing to alarming proportions in several parts of the world (WHO, 1998). The major concerns are the co-morbidities associated with obesity such as type 2 diabetes mellitus, cardiovascular disease, stroke and certain types of cancers, as well as the predictors (risk factors) of these diseases, including insulin resistance, dyslipidaemia and hypertension, in other words, the metabolic syndrome.

There is now strong evidence for an established difference between central (abdominal, visceral) and peripheral (gluteofemoral) obesity in the associations to these diseases. Central obesity shows most of the hazardous complications in a number of cross-sectional and prospective studies (for review see Björntorp, 1999a). Central obesity is most prevalent among men and post-menopausal women, and might contribute to the high incidence of cardiovascular disease in these groups. In moderate degrees, peripheral obesity appears to be mainly a cosmetic problem. It is important to realize these differences in risks when the growing problems with obesity are considered (Björntorp, $1999 a)$.

Central obesity is consequently the condition where the metabolic syndrome is most frequently found. The pathogenesis for this combination of symptoms is not known. This problem is of major importance because it may hide the background to prevalent disease.

\section{Cortisol secretion in obesity}

Clinicians are struck by the immediately apparent differences between central and peripheral obesity, and systematic descriptions appeared more than half a century ago (Kretschmer, 1921; Vague, 1947). Central obesity is 
sometimes described as a 'Cushingoid' form of obesity because of the apparent similarities with Cushing's syndrome. Therefore numerous studies have been performed on cortisol secretion in obesity. The results have shown, in short, that obesity is characterized by an increased production of cortisol, combined with an elevated fractional turnover rate, which results in normal or lower-than-normal circulating cortisol. The feedback control of the hypothalamic-pituitary adrenal (HPA) axis has been found to be normal, utilizing the conventional $1 \mathrm{mg}$ dose of dexamethasone (Björntorp, 1999b).

Unfortunately, these studies have usually not separated central and peripheral obesities, which would have been desired (or even expected) knowing the difference in external appearance of these both conditions: in fact only central obesity should have been suspected to show pathological features of cortisol secretion. There are, however, studies where the features of cortisol secretion found in obesity in general seem to be more pronounced in central than in peripheral obesity (Björntorp, 1999b).

A common problem with these studies seems, however, to be that the examinations performed have often been those conventionally used in the clinical diagnosis of Cushing's syndrome. Such methods are probably too insensitive, with little discriminative power for milder forms of HPA axis hyperactivity. These tests are also usually performed with the proband in the unfamiliar milieu of a hospital or laboratory and include venipunctures, both factors being likely to disturb the sensitive regulatory system to be examined. Strain-causing events may occur under everyday life conditions, resulting when frequently repeated in damaging long-term consequences. Cortisol secretion following such events is only discoverable in the natural milieu. In turn, this means that sampling procedures must be employed that are not dependent on a laboratory setting.

Cortisol in saliva meets such requirements because of the possibility of delivering repeated saliva samples under most circumstances except during sleep. Furthermore, saliva cortisol measures free, circulating cortisol, and is independent of changes in saliva secretion (Kirschbaum \& Hellhammer, 1994). Measurements of cortisol in saliva seem to be ideal for the requirements needed, and are relevant for the purpose of examining minor perturbations of cortisol secretion in everyday life.

The usefulness of this methodology for the purpose in question has recently been beautifully documented in a study of normal subjects (Smyth et al. 1998). The probands delivered saliva for cortisol measurements on six random time points over an ordinary working day, and reported simultaneously the mood and environmental pressures, perceived at the time of sampling, due to ongoing, anticipated or previously experienced problems. With these procedures it was possible to demonstrate the dramatic influences of such factors on cortisol secretion, which varied in a 'doseresponse'-dependent manner up to 20 -fold over a day. Not only previous, current and anticipated environmental factors had an influence on cortisol concentrations, but also moods: a happy mood was followed by low cortisol, while feeling miserable or angry had the opposite effect. This study demonstrates the dramatic flexibility and sensitivity of the HPA axis even during an ordinary working day.

\section{Recent studies employing saliva cortisol}

We have used saliva cortisols to study the potential association between cortisol and the metabolic syndrome. These studies have been performed on a random population basis in both middle-aged men (52 years) and women (40 years). Only the results for the men are currently available, and will be summarized in the following (Rosmond et al. 1998, 1999a).

Saliva was collected repeatedly over an ordinary working day, with perceived stress self-registered each hour from morning to bed-time. This allowed examinations of the kinetics of HPA axis activity including morning and evening concentrations, and total cortisol secretion over the day. In addition, the response to a standardized lunch and stress-related cortisol were recorded, as well as the suppression by a low dose $(0.5 \mathrm{mg})$ of dexamethasone. These measurements were then set in relation to anthropometric registrations (BMI; waist-hip ratio, WHR; and abdominal sagittal diameter), determinations of overnight fasting values of testosterone and insulin-like growth factor I (a substitute for growth hormone secretion), metabolic variables (glucose, insulin, triglycerides, total, LDL and HDL cholesterol), as well as blood pressures and heart rate.

\section{Results and discussion}

\section{Characteristics of diurnal curves}

As expected, the diurnal cortisol curves showed a continuous spread of saliva cortisol concentrations. These results were not possible to sort out in a meaningful way in curves of different, defined characteristics, even with arbitrarily chosen borderlines. To avoid these difficulties we therefore used a measurement of cortisol variability over the day which depends mainly on the differences between morning (high) and evening (low) cortisol values. By a statistical weighting procedure (Rosmond et al. 1998) of high or low variability, it was possible to visualize two types of diurnal curves (Fig. 1). One had high morning and low evening values, and was considered to represent a normally functioning HPA axis. Another type of curve was flat and showed low morning values, not much different from evening concentrations, probably a pathological condition.

The use of the weighting procedure made it possible to sort out these different day curves, but does not allow quantification of the prevalence of the normal and pathological cortisol secretion. This procedure utilizes all measurements from the men with a different weight of the analysed variable and is exaggerating the difference. The variability of the measured cortisols, used as a weighted separation characteristic, is dependent mainly on the concentration of morning cortisols, because the evening cortisols were not much different between the two curve types (Fig. 1). Morning cortisol may therefore be used as a surrogate measurement of diurnal variability, and is less complex to handle to define arbitrary cut-off points. This procedure also seems justified by the high correlation between variability and morning cortisol $(r=0 \cdot 870)$.

The average morning saliva cortisol was $14.7 \pm 7.2$ (SD) $\mathrm{nmol} / \mathrm{l}$. The values appear normally distributed. There were 12 men with values $<5 \mathrm{nmol} / \mathrm{l}$, and 47 men with between 5 


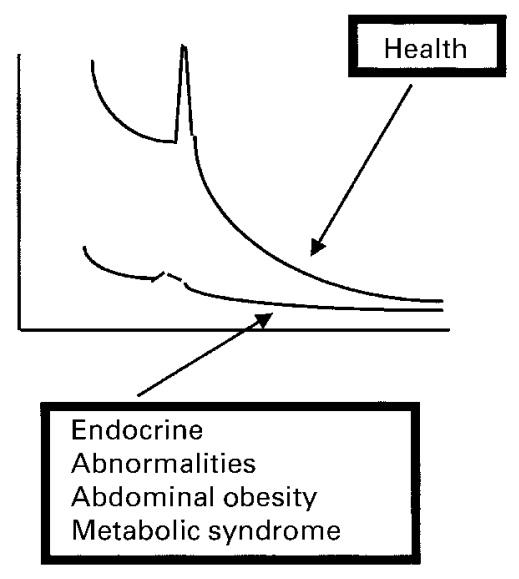

Fig. 1. Schematic representation of normal diurnal cortisol secretion with high morning and low evening values, with a peak after lunch, associated with health (upper curve), and a pathological secretion with low morning and lunch values, associated with other endocrine abnormalities (low testosterone and growth hormone values), abdominal obesity and the metabolic syndrome. In this condition there are also signs of elevated activity of the sympathetic nervous system. Curves obtained by a statistical weighting procedure (from Rosmond et al. 1998).

and $<10 \mathrm{nmol} / \mathrm{l}$. If an arbitrary cut-off point is set at the first concentration, less than $5 \%$ had abnormal values. If instead $<10 \mathrm{nmol} / 1$ is chosen as a cut-off point, then about $20 \%$ had abnormal cortisol secretion. Although the selection of borderline is totally arbitrary, such calculations suggest that a pathological cortisol secretion was found in a small number of the men examined.

\section{Stress-related cortisol}

Stress-related cortisol secretion was calculated as a coefficient of co-variability between all cortisol values and corresponding stress reports over the day. Stress was registered as a yes/no variable. A high stress-related cortisol value was due to high cortisol values on the occasions when stress was reported. Similarly, low stress-related cortisol secretion indicates low cortisol in relation to reported stress. The division line between high and low stress-related cortisol was set where perceived stress had no measurable effect on cortisol values.

It should be observed that this method of calculation does not provide information on the occasion, severity or duration of the perceived stress. Since it measures cortisol concentrations in relation to perceived stress on several registered occasions over a day, this method provides an indication of the sensitivity of the system to react to perceived stress with elevated cortisol secretion. Calculated in this way, about $39 \%$ of the men responded to perceived stress with elevated cortisol secretion to a varying degree.

\section{Subgrouping}

Firstly, groups of men with normal HPA axis regulation (as defined in the previous section; Fig. 1, upper curve) and high or low stress-related cortisol, were separated out, and the absolute saliva cortisol values over the day calculated. As

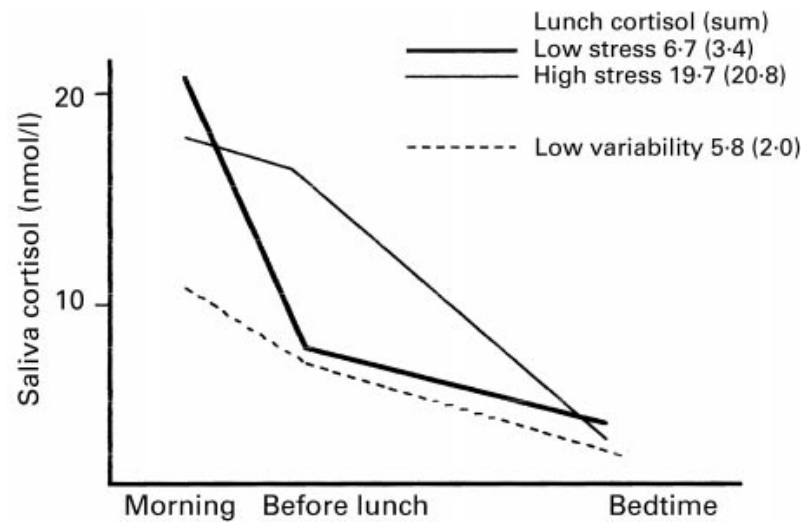

Fig. 2. Cortisol values in the morning, before lunch (sum of lunch cortisol) and in the evening in men with a normal hypothalamicpituitary adrenal (HPA) axis function (high morning, low evening cortisols) with high (thin line) and low (thick line) stress-related cortisol, and in men with a pathological HPA axis function (low variability; broken line). Sum of all cortisol values at 30, 45 and $60 \mathrm{~min}$ after a standardized lunch. Statistically significant comparisons with the men with normal HPA axis: morning, high stress, $P=$ 0.004 , low variability, $P<0.001$; before lunch, high stress, $P<0.001$; lunch, high stress, $P<0.001$, low variability, $P<0.05$ (from Rosmond et al. 1998, 1999a, and unpublished results).

seen in Fig. 2, men with a normal HPA axis regulation and high-stress-related cortisol secretion had significantly higher total cortisol secretion than men with a normal axis and low stress-related cortisol secretion. The difference was due to an apparent inability of the high-stress men to "wind down' their cortisol values during the morning hours before lunch, plus a larger food-induced cortisol secretion measured during the standardized lunch. Evening values were, however, not different from men reporting low stress.

A similar subdivision of the men with pathological HPA axis function (Fig. 1, lower curve) showed no striking differences in cortisol values with low or high stress-related cortisol secretion (not shown).

This subdivision of arbitrary groups of men thus yielded three groups: men with normal kinetics of the diurnal cortisol curve (high morning, low evening cortisols; Fig. 1); with or without elevated stress-related cortisol; and with low cortisol secretion (Fig. 2). The latter group was not subdivided because of apparent small or no differences in cortisol secretion in groups of high and low reported stress.

We have interpreted these findings as follows. Men with a normal HPA axis regulation without much cortisol secretion related to everyday stress have been considered as a normal reference group. In men with stress sensitivity, who still have a normally functioning HPA axis, cortisol secretion becomes elevated over the day, particularly in the measurements before noon. Food intake is also associated with an elevated cortisol response, indicating an increased sensitivity of cortisol secretion to a standardized, physiological stimulus. Taken together, these measurements suggest a frequently activated and sensitized regulatory system of the HPA axis in the group of men where a normal HPA axis function is exposed to stress, with elevated cortisol secretion as a consequence. This group constituted about one-third of the population. 
In a small part of the material, approximately $10 \%$, the cortisol concentrations over the day are clearly different, showing little variability over the day, not much reaction to perceived stress, and less stimulation by feeding than in the reference group. Total diurnal cortisol secretion was about $75 \%$ of that in the group with a normal HPA axis with low stress-related cortisol, and the diminution was due mainly to low morning values and to a somewhat lower response to lunch. Such a 'rigid' diurnal curve with low plasticity has previously been observed after prolonged severe stress, such as in war veterans, chronic pain and in circumstances of being 'cornered' in a seemingly irreversible poor environment, causing 'vital exhaustion', a so-called helplessness reaction (McEwen, 1998).

\section{Comparisons with animal studies}

The observations summarized above are so far cross-sectional. Results of detailed, controlled intervention studies in animals are helpful for interpretation of the human data. In rats which are chronically stressed, an early consequence is a facilitation of the activity of the HPA axis during the phases where the HPA axis is endogenously most active (Dallman et al. 1992). This might correspond to our observations of an increased cortisol secretion during the prenoon hours and during lunch in men who report stress involvement, because during this period the HPA axis is most active in humans. When stress is prolonged the repeated cortisol peaks during occasions of stress cause elevated cortisol secretion, which eventually is followed by a decreased 'winding down' of the stress-induced cortisol peaks, followed by a decreased, rigid cortisol secretion, a 'burn-out', where low morning cortisols are a hallmark. At this stage sympathetic nervous sytem activity is increasing, which might be considered as a compensatory phenomenon to maintain homeostasis or allostasis in various somatic systems (McEwen, 1998). This development might be what we are seeing in the results of studies in humans, with first a stress-induced increased cortisol secretion, which then might be followed by a burn-out condition with low diurnal cortisol secretion. This might be considered as a 'Starling curve' of the HPA axis. By follow-up studies we intend to examine these phenomena as far as possible in humans, but we have already seen consistent statistical correlations between the supposed stages of HPA axis activity derangements and factors which are known or suspected to increase HPA axis activity, such as psychosocial and socioeconomic handicaps, alcohol consumption, smoking, and traits of depression and anxiety (Björntorp, 1993, 1999a).

\section{Associations between HPA axis activity and somatic variables}

The somatic variables were then compared between these groups (Rosmond et al. 1998, 1999a). Several striking differences were then found in correlation analyses. In men with a normal diurnal cortisol curve and low stressrelated cortisol basal, non-stimulated cortisol secretion was negatively related to anthropometric, metabolic and hemodynamic values. This was particularly clear when examined in relation to lunch cortisols (Table 1). In contrast, in the men with normal diurnal kinetics of cortisol values, but with high reported stress, resulting in elevated total (particularly pre-noon and lunch) cortisols, positive relationships were found between cortisol values and sagittal, abdominal diameter and metabolic variables, indicating the metabolic syndrome. Furthermore, diastolic blood pressure was elevated in relation to cortisol secretion (Table 1 ).

We interpret these results as follows. The group of men with normal diurnal cortisol values (high morning, low evening concentrations) and low sensitivity to stress, resulting in normal cortisol secretion over the day, represents men with an intact regulatory system for the HPA axis, with little influence by diurnal stressors. This group then has been considered to represent a normal condition, and constitutes about two-thirds of the population. Such a normal HPA axis regulation of cortisol secretion, not much influenced by stress, is apparently proportional to healthy somatic variables.

The group with apparently normally regulated cortisol secretion with high morning and low evening values, but a sensitization to perceived stress with elevated total cortisol values, showed positive relationships particularly to anthropometric and metabolic variables. Here cortisol might be

Table 1. Relations between indicated variables and cortisol secretion in men with normal and pathological function of the hypothalamic-pituitary adrenal axis (HPA) in the basal state after lunch and in relation to reports of perceived stress in 284 randomly selected 51 -year-old men

\begin{tabular}{|c|c|c|c|c|c|c|}
\hline \multirow[b]{2}{*}{ Variables } & \multicolumn{3}{|c|}{ Normal HPA } & \multicolumn{3}{|c|}{ Pathological HPA } \\
\hline & Basal & Lunch & Stress & Basal & Lunch & Stress \\
\hline Anthropometric & - & --- & ++ & $(-)$ & +++ & +++ \\
\hline Endocrine & + & & + & & - & --- \\
\hline Insulin & & --- & ++ & ++ & +++ & +++ \\
\hline Insulin/glucose ratio & & --- & ++ & ++ & +++ & +++ \\
\hline Lipids & & --- & ++ & -- & +++ & +++ \\
\hline Hemodynamic & - & --- & $(+)$ & +++ & +++ & +++ \\
\hline
\end{tabular}

Anthropometric variables: BMI, waist-hip circumference ratio, abdominal, sagittal diameter.

Endocrine variables: testosterone, insulin-like growth factor I.

Lipids: triglycerides, total LDL and HDL cholesterol.

Hemodynamic variables: systolic and diastolic blood pressures and heart rate

Signs indicate the direction and approximate strength of significant correlations: low HDL indicated by positive labels.

Source: Rosmond et al. 1998, 1999a, and unpublished results. 
considered to be the trigger, particularly when challenges of various nature stimulated a sensitized system, including food intake. Whether or not nocturnal cortisol secretion contributed to a difference in total cortisol secretion was not measured.

In the men with low, pathological cortisol secretion there were consistent, strongly positive relationships to measurement indicating central obesity (BMI, WHR and sagittal diameter), insulin, glucose, lipids (HDL, cholesterol negative), systolic and diastolic blood pressures and heart rate. This was found both with stress-related cortisol and with cortisol secretion after lunch, and is a full-blown picture of the metabolic syndrome including central obesity and elevated blood pressure (Table 1). We have interpreted this to mean that a pathologically regulated cortisol secretion is strongly related to somatic abnormalities, particularly visible after HPA axis challenges (stress or lunch). In this condition, however, total cortisol secretion is not elevated and is unlikely to be directly responsible for the peripheral abnormalities.

\section{Involvement of sex steroid and growth hormones and the sympathetic nervous system}

In the situation with a pathological HPA axis regulation, other potential causes than elevated cortisol secretion for the associated somatic perturbations have to be sought. Two possibilities seem apparent from the results obtained. Firstly, testosterone and IGF-I are consistently low in this condition (Table 1), and may be at least partly responsible for the appearance of the metabolic syndrome. This possibility seems likely in the face of observations of effects of these hormones in balancing cortisol effects on fat distribution and metabolism. Furthermore, substitutions with these hormones are followed by marked improvements of the metabolic syndrome, including visceral fat mass (Björntorp, 1993, 1966). It should be noted that perturbations of the regulation of the HPA axis are followed by inhibitions of the central axes for growth and sex steroid hormone secretion (Chrousos \& Gold, 1992); in other words, HPA axis disturbances might be the primary factor for abnormalities in the gonadal and growth hormone axes.

An additional possibility might be that the central sympathetic nervous system seems to be activated in this condition of a pathological, burned-out HPA axis. This possibility is indicated by the robust associations with elevated blood pressure and heart rate (Table 1). This might correspond to observations in controlled animal experiments where a faltering HPA axis activity is followed by elevated activity of the sympathetic nervous system, perhaps as a protective compensation to maintain homeostatic or allostatic conditions (Calogero et al. 1988). Such activation has been considered as the trigger for the development of hypertension in the early hyperkinetic state (Folkow, 1993).

Another consequence of elevated sympathetic nervous system activity would be expected to be a stimulation of mobilization of free fatty acids. Elevated circulating free fatty acid concentration is a well established phenomenon in central obesity (Kissebah et al. 1998). Although this was not measured in the work discussed here, the men with signs of elevated sympathetic nervous activity were also clearly centrally obese. Elevated free fatty acids induce insulin resistance in muscle (Randle et al. 1963) and provide substrate for hepatic production of lipoproteins (Björntorp, 1990). Elevated free fatty acids would therefore be expected to be responsible for or amplify the metabolic perturbations of the men in this subgroup with pathological regulation of the HPA axis.

A better understanding is needed of the mechanisms for the severe somatic perturbations of the subgroup of men with a pathological but low total cortisol secretion. The strong relationships to an abnormal regulation of HPA axis function rather than total cortisol secretion suggest that the pathogenesis is in some way associated with neuroendocrine perturbations. The low sex steroid and growth hormone secretions, as well as increased activity of the sympathetic nervous system, may well be other signs of central regulatory abnormalities which can be derived from primary aberrations in HPA axis regulation. There are probably additional pathogenetic pathways. Although this subgroup of pathological HPA axis regulation is limited in the population (approximately $10 \%$ ), the powerful associations to a broad risk-factor pattern of disease make it important to understand this condition.

In summary, the metabolic syndrome appears under two of the conditions of HPA axis activity examined. The first is with elevated, stress-related cortisol secretion, and the second with a perturbed HPA axis function without elevated cortisol secretion, a situation with a burn-out of the HPA axis. It is presumed that in the first case cortisol is responsible for the pathogenesis of the metabolic syndrome. For the burn-out, low sex steroid and growth hormone secretions combined with an elevated activity of the central sympathetic nervous system might generate the metabolic syndrome. The condition of elevated stress-related cortisol secretion appears to occur in about $20-25 \%$ of the men examined, and the pathological HPA axis function in some $10 \%$ in approximate, arbitrary figures. This does not mean, however, that a total of 30-35\% of the men suffer from the metabolic syndrome, because the associations between HPA axis perturbations and the metabolic syndrome are statistical phenomena. We have abstained from drawing further arbitrary borderlines with the ambition of giving prevalence figures for the metabolic syndrome. However, it seems likely that a large fraction of the metabolic syndrome may be recruited via these neuroendocrine pathways, affecting central axes of both endocrine regulations and the sympathetic nervous system.

The combined appearance of signs of activation of several neuroendocrine pathways is an expected phenomenon, and is probably due to the tight functional associations of the regulatory centres at several levels. In fact it is difficult to activate one isolated pathway to the periphery: usually combined responses to challenges are occurring along both the HPA axis and the sympathetic nervous system, a 'hypothalamic arousal'. When this progresses for a sufficiently long period, the gonadal, growth hormone and thyroid axes become secondarily inhibited (Chrousos \& Gold, 1992). Such combined perturbations would be expected to affect several peripheral somatic systems in a powerful way, probably including the pathogenesis of the metabolic syndrome. 


\section{Genetic aspects}

\section{Polymorphisms of genes involved in HPA axis regulation}

The metabolic syndrome is probably based on a number of predisposing genetic factors. Each component in this cluster of symptoms might be based on polymorphisms of genes in peripheral tissues regulating visceral fat accumulation, insulin sensitivity, dyslipidaemia and blood pressure. Efforts to find such abnormalities have so far apparently not been very successful (Bouchard \& Perusse, 1996). Having seen the possibility of generating somatic perturbations by central regulatory neuroendocrine errors, we have focused on central regulatory candidate genes. It has been reported, for example, that cortisol secretion is strongly genetically regulated: homozygotic twins show almost identical diurnal HPA axis activity (Linkowski et al. 1993).

The feedback control of the HPA axis seems to be mildly deficient in the metabolic syndrome, visible with suppression of cortisol secretion with low doses of dexamethasone (Ljung et al. 1996; Rosmond et al. 1998). Preliminary functional tests suggest that the sensitivity of the central glucocorticoid receptors (GR) is diminished, which might be due to a low density of these GRs. This could be a secondary phenomenon by down-regulation of GR density due to frequent HPA axis challenges, which under severe conditions, such as Cushing's syndrome or melancholic depression, finally results in irreversible loss of brain substance in the areas of regulating GRs (McEwen, 1998). There is of course also the possibility that such deficient regulation of central GR density might be due to a genetic susceptibility. A suspected area for such an error would then be in the regulatory domain of the GR gene locus.

Studies using restriction fragment length polymorphism (RFLP) have revealed some potentially interesting associations along these lines, again utilizing the material of men described in the preceding sections. An advantage of this approach is the detailed phylogenetic characteristics of this material, and the likelihood that it represents the population at large.

A polymorphism is found with the restriction enzyme $B c l \mathrm{I}$, localized to the first intron of the GR gene. This is associated with poor control of cortisol secretion after feeding (Fig. 2; Table 2) (Rosmond et al. 1999b), as well as abdominal obesity, insulin resistance and elevated blood pressure (Weaver et al. 1992; Watts et al. 1992; Buemann et al. 1997; Rosmond et al. 1999b). About $14 \%$ of the men are homozygotes for this polymorphism. Its localization in a non-coding area of the gene does not exclude a potential functional importance such as perturbed splicing of primary mRNA. This polymorphism might, alternatively, indicate a functionally important polymorphism in the vicinity.

An interesting newly discovered phenomenon in steroid hormone genes is the appearance of trinucleotide repeats (CAG). Such repeats in early exons interfere in a quantitative manner with transcription of steroid hormone receptor genes (Riggins et al. 1992). The first exon of the GR gene is silent. Sequencing the second exon has not revealed abnormal numbers of CAG repeats in men with the metabolic syndrome in comparison with men without metabolic abnormalities or a group of normal subjects in the laboratory (M. Ottosson et al., unpublished results).
Finally, a polymorphism has been localized to the $5^{\prime}$ flanking region of the GR gene locus, utilizing the restriction enzyme TthIII-1. This is associated with elevated basal cortisol secretion and is found in $8 \%$ (homozygotes) of the men. The relationship to metabolic and other variables is uncertain (R. Rosmond et al., unpublished results). However, this finding suggests the potential involvement of polymorphisms of the promotor region of the GR gene, and we are currently sequencing the first part of this domain.

It should be noted in this context that the preliminary results of the studies in this sector indicate that the sensitivity of the central feedback control of the HPA axis appears to be diminished in functional studies. This is in accordance with the findings of an elevated sensitivity of the HPA axis upon perceived stress and feeding. Such a diminished sensitivity would have the potential to explain many of the phenomena observed, and is therefore important to pursue. The preliminary findings of associations of polymorphisms of the GR gene with HPA axis sensitivity seem to point in the same direction, possibly affecting regulation of GR density and therefore HPA axis sensitivity. A potential functional importance of these polymorphisms therefore has a high priority for further studies.

\section{Polymorphisms of genes involved in the regulation of the sympathetic nervous system}

There are also potentially interesting polymorphisms in the regulatory pathways of the sympathetic nervous system. Dopamine receptors are involved in blood pressure regulation via salt retention (dopamine receptor 1) or by controlling noradrenaline outflow from nerve endings (dopamine receptor 2) (Missale et al. 1998). The latter receptor shows a polymorphism which is strongly associated with elevated blood pressure (R. Rosmond et al., unpublished results). If functionally important, such a polymorphism would be expected to increase the sensitivity of signals to regulatory centres of the sympathetic nervous system and thereby increase blood pressure. This is of potential interest in view of the involvement of the sympathetic nervous system in the metabolic syndrome and in early hyperkinetic hypertension (Folkow, 1993).

The leptin receptor shows polymorphisms which are also associated with blood pressure regulation, but in an opposite way. Leptin has recently been found in animal experiments to stimulate the central sympathetic nervous system, resulting in elevated blood pressure. This is accomplished via signals from the leptin receptor. With mutations of the

Table 2. Saliva cortisol after lunch in relation to a $B c / l$ polymorphism of the glucocorticoid receptor (GR) gene locus (from Rosmond et al. 1999b)

\begin{tabular}{lccc}
\hline & $\begin{array}{c}\text { Homozygotes } \\
(n=30)\end{array}$ & $\begin{array}{c}\text { Wild type } \\
(n=89)\end{array}$ & $\begin{array}{c}\text { Heterozygotes } \\
(n=99)\end{array}$ \\
\hline $30^{\prime}$ & $11 \cdot 1^{* *}$ & $7 \cdot 1$ & $8 \cdot 6\left(^{\star}\right)$ \\
$45^{\prime}$ & $9 \cdot 2^{\star * *}$ & $6 \cdot 4$ & $8 \cdot 0^{\star *}$ \\
$60^{\prime}$ & $10 \cdot 0^{* *}$ & $5 \cdot 8$ & $7 \cdot 3^{\star *}$ \\
Total peak (nmol/l) & $10 \cdot 1^{* *}$ & $6 \cdot 4$ & $7 \cdot 9^{*}$ \\
\hline
\end{tabular}


receptor gene in rodent models resulting in a non-functioning protein, blood pressure is not elevated despite severe obesity and large elevations of both leptin and insulin (Haynes et al. 1997; Dunbar et al. 1997).

In the men we have studied, polymorphisms of the leptin receptor gene locus are associated with considerably lower blood pressure. Men with elevated blood pressure appear to be essentially free of this polymorphism. Furthermore, men with abdominal obesity and elevated leptin and insulin levels are apparently protected from hypertension if the leptin receptor is polymorphic ( $\mathrm{R}$. Rosmond et al., unpublished results).

In summary, the regulatory systems of both the major centres for neuroendocrine-endocrine and autonomic nervous system signals to the periphery show polymorphic genes in RFLP studies. Further studies along these lines may lead to a better understanding of the genetic basis of the metabolic syndrome and primary hypertension. It is anticipated that sensitivity to environmental pressures would be more pronounced in the presence of functionally significant polymorphisms in the central regulatory systems for neuroendocrine secretions and the autonomic nervous system.

\section{Environmental factors}

The centres for both the regulation of the HPA axis and the sympathetic nervous system are susceptible to the influence of a number of environmental factors. The function of these centres is to serve as a buffer against such environmental pressures through allostatic mechanisms (McEwen, 1998). However, when such inputs become intensive, often repeated or prolonged, these protective mechanisms may be damaging.

Environmental challenges of this type are frequently collected under the heading 'stress'. Under this collective label are included a number of factors from psychological pressures to toxins, diseases, depression, anxiety, pain, etc. Among psychological factors, handicaps in psychosocial and socio-economic conditions would be expected to provide a basis for perception of anxiety, loneliness, boredom, a limited latitude for one's own decisions, monotonous, poorly paid work due to low education, etc. All these factors have been found to be associated to perturbed HPA axis function in population studies, with some gender profile in relation to the impact on neuroendocrine regulations. For example, men are more vulnerable than women as far as psychosocial (divorce and living alone) and socio-economic factors (problems at work and unemployment) (Björntorp, 1999a; Björntorp et al. 1999). We suggest that such factors initiate frequent challenges of the allostatic mechanisms, leading to peripheral disease-generating mechanisms in the long run.

At least two types of observation indicate that this is a correct interpretation. The Whitehall studies (Brunner et al. 1997) have recently reported that a negative socio-economic gradient is associated with abdominal obesity and the metabolic syndrome, confirming previous studies (Björntorp, 1993). We find that a similar negative gradient is associated not only with central accumulation of body fat mass, but also with HPA axis perturbations, elevated stress-related cortisol secretion, and a diminished dexamethasone suppression. In addition, exposure time for poor socio-economic status seems to worsen these associations (Rosmond \& Björntorp, 1999). These findings might provide a mechanism for the 'social inequality of disease' (Brunner et al. 1997).

Furthermore, fully controlled experiments in primates show that exposure to moderate psychosocial stress (change of hierarchy in a colony) is followed by a depressive psychological reaction, enlarged adrenals, a diminished suppressibility of the HPA axis activity by low-dose dexamethasone, decreased secretion of sex steroid hormones, and a full-blown metabolic syndrome, including visceral fat accumulation, insulin resistance, dyslipidaemia, elevated blood pressure, and early symptoms of type 2 diabetes (impaired glucose tolerance) and coronary artery atherosclerosis (Shively et al. 1997). This is what we find in humans exposed to psychosocial and other strains. The similarities strongly suggest a cause-effect relationship.

\section{Intervention studies}

Direct intervention studies are informative in determining cause-effect relationships. We have performed several such studies with replacements of sex steroid and growth hormones (Björntorp, 1993). Convincing global improvements have been obtained, suggesting that the involvement of a diminished secretion of these hormones is of importance, as discussed above.

Direct interventions against the HPA axis perturbations are trickier, for several reasons. Firstly, the mixed picture of involvement of this axis makes interventions complicated. We have, for example, tried a GR antagonist (RU 486, Roussel UCLAF, Paris) with the aim of blocking the peripheral GR without much success, perhaps due to a lack of specificity, or a breakthrough of an endogenous, compensatory increase of cortisol secretion, or perhaps because we studied a mixed bag of HPA perturbations not known at the time these studies were performed (unpublished results). The danger of side effects by interference with this vitally important system is a major concern in trials with agents interfering with cortisol metabolism.

A different approach is to try interventions at levels proximal to the centres regulating the neuroendocrine axes. There are several features of the cascade of events leading to the neuroendocrine pathogenesis of the metabolic syndrome, which suggest involvement of the central serotonergic system. This system is known to regulate the HPA axis, and is involved in depression, anxiety, alcohol consumption and carbohydrate craving (Eriksson \& Humble, 1990), all symptoms we have found to be involved in the neuroendocrine background to the metabolic syndrome. We have therefore recently finalized a pilot study with a trial of a serotonin reuptake inhibitor (Cipramil, Lundbeck, Copenhagen, Denmark) that does not influence appetite regulation, which would prevent conclusions due to the confounding effect of body weight decrease. This resulted in signs of improvement in both sympathetic nervous system and HPA axis activities, with some improvements also in metabolic variables. These were men without much symptom of depressive mood traits, and no significant changes occurred in such variables (unpublished results). 
This might mean that certain antidepressants act on neuroendocrine regulation of metabolism separately from their antidepressant action. Although a short-term study with relatively few subjects involved $(n=15)$, these preliminary results encourage further attempts in this direction which are now being planned.

\section{Concluding remarks}

This review summarizes neuroendocrine abnormalities associated with the metabolic syndrome. Focus has been placed on cortisol and the regulation of the HPA axis. The results emerging show a complex picture, where perturbed HPA axis regulations appear to be at least equally as important as raised cortisol levels in the presumed pathogenesis of the metabolic syndrome. This is indicated by the negative associations between cortisol secretion and somatic variables in the group with a normal HPA axis function, not much affected by stressors, where the kinetics rather than absolute values of cortisol secretion seem to relate to somatic health. Furthermore, the results in the subgroup with severely deranged HPA axis function with low total cortisol secretion visualize this possibility particularly clearly. Here again the kinetics of cortisol secretion, which is showing signs of a burn-out, is strongly associated with somatic abnormalities, while cortisol secretion is low. In this situation there is evidence of the influence of other neuroendocrine and autonomic systems, and it may be considered that the peripheral perturbations of endocrine, anthropometric, metabolic and hemodynamic systems are consequences of complex, central, integrated disturbances. One might predict that the leptin system and more proximal regulator systems are also involved, although the regulation of the HPA axis may occupy a central position. This field offers a challenge for further studies.

\section{References}

Björntorp P (1990) 'Portal' adipose tissue as a generator of risk factors for cardiovascular disease and diabetes. Arteriosclerosis 10, 493-496.

Björntorp P (1993) Visceral obesity: a 'Civilization Syndrome'. Obesity Research 1, 216-222.

Björntorp P (1996) The regulation of adipose tissue distribution in humans. International Journal of Obesity and Related Metabolic Disorders 20, 291-302.

Björntorp P (1999a) Overweight is risking fate. Best Practice and Research in Clinical Endocrinology and Metabolism 13, 47-69.

Björntorp P (1999b) Neuroendocrine perturbations as a cause of insulin resistance. Diabetes/Metabolism Research and Reviews, in press.

Björntorp P, Holm G \& Rosmond R (1999) Hypothalamic arousal, insulin resistance and type 2 diabetes mellitus. Diabetic Medicine 16, 373-383.

Bouchard C \& Perusse L (1996) Current status of the human obesity gene map. Obesity Research 4, 81-90.

Brunner EJ, Marmot MG, Nanchahal K, Shipley MJ, Stansfield SA, Juneja M \& Alberti KGMM (1997) Social inequality in coronary risk: central obesity and the metabolic syndrome. Evidence from the Whitehall II study. Diabetologia 10, 13411349.

Buemann B, Vohl M-C, Chagnon M, Chagnon YC, Gagnon J, Perusse L, Dionne F, Despres J-P, Tremblay A, Nadeau A \& Bouchard C (1997) Abdominal visceral fat is associated with a
$B c l$ I restriction fragment length polymorphism at the glucocorticoid receptor gene locus. Obesity Research 5, 186-192.

Calogero AE, Callucci WT, Gold PW \& Chrousos GP (1998) Multiple regulatory feedback loops on hypothalamic-corticotropin releasing hormone secretion. Journal of Clinical Investigation 82, 76-98.

Chrousos GP \& Gold PW (1992) The concept of stress and stress system disorders: overview of physical and behavioral homeostasis. Journal of the American Medical Association 267, 12441252.

Dallman MF, Akana SF, Scribner KA, Bradbury MJ, Walker C-D, Strack AM \& Cascio CS (1992) Stress, feedback and facilitation in the hypothalamo-pituitary adrenal axis. Journal of Neuroendocrinology 4, 517-526.

Dunbar JC, Hu Y \& Lu H (1997) Intracerebroventricular leptin increases lumbar and renal nerve activity and blood pressure in normal rats. Diabetes 46, 2040-2045.

Eriksson E \& Humble M (1990) Serotonin in psychiatric pathophysiology. The Biological Basis for Psychopharmacological Treatment. Progress in Basic and Clinical Pharmacology, pp. 66-119 [R Pohl and S Gerson, editors]. Basel, Switzerland: Karger.

Folkow B (1993) Physiological organization of neurohormonal responses to psychosocial stimuli. Implications for health and disease. Annals of Behavioural Medicine 15, 236-244.

Haynes WG, Morgan DA, Walsh SA, Mark AL \& Sivitz WI (1997) Receptor mediated regional sympathetic nerve activation by leptin. Journal of Clinical Investigation 100, 270-278.

Kirschbaum C \& Hellhammer DH (1994) Salivary cortisol in psychoneuroendocrine research: recent developments and applications. Psychoneuroendocrinology 19, 313-333.

Kissebah AH, Krakower GR, Sonnenberg G \& Hermes MMI (1998) Clinical manifestations of the metabolic syndrome. In Handbook of Obesity, pp. 601-636 [GA Bray, C Bouchard \& CPT James, editors]. New York: Marcel Dekker.

Kretschmer E (1921) Körperbau und Character, 1st edn. Berlin: Springer (1967, 25th edn).

Linkowski P, Van Onderbergen A, Kerkhofs M, Bosson D, Mendlewics J \& Van Cauter E (1993) Twin study of 24-h cortisol profile: evidence for genetic control of the human circadian clock. American Journal of Physiology 264, 173-181.

Ljung T, Andersson B, Bengtsson B-Å, Björntorp P \& Mårin P (1996) Inhibition of cortisol secretion by dexamethasone in relation to body fat distribution: a dose-response study. Obesity Research 4, 277-282.

McEwen BS (1998) Protective and damaging effects of stress mediators. New England Journal of Medicine 338, 171-179.

Missale C, Russel Nash S, Robinson SW, Jaber M \& Caron MG (1998) Dopamine receptors: from structure to function. Physiological Reviews 78, 189-122.

Randle P, Garland P, Hales N \& Newsholme E (1963) The glucose fatty acid cycle: its role in insulin sensitivity and the metabolic disturbances of diabetes mellitus. Lancet 785-789.

Reaven GM (1988) Role of insulin resistance in human disease. Diabetes 37, 1595-1607.

Riggins GJ, Lokey KL, Chastain JL, Leiner HA, Sherman SL, Wilkinson KD \& Warren ST (1992) Human genes containing trinucleotide repeats. Nature Genetics 2, 186-191.

Rosmond R \& Björntorp P (1999) Occupational status, cortisol secretory pattern and visceral obesity in middle-aged men. Journal of Internal Medicine, in press.

Rosmond R, Dallman MF \& Björntorp P (1998) Stress-related cortisol secretion in men: relationships with abdominal obesity and endocrine, metabolic and hemodynamic abnormalities. Journal of Clinical Endocrinology and Metabolism 83, 853-859.

Rosmond R, Holm G \& Björntorp P (1999a) Food-induced cortisol secretion in relation to anthropometric, metabolic and 
hemodynamic variables in men. International Journal of Obesity and Related Metabolic Disorders, in press.

Rosmond R, Chagnon YC, Holm G, Chagnon M, Perusse L, Carlsson B, Bouchard C \& Björntorp P (1999b) A Bcl I restriction fragment length polymorphism of the glucocorticoid receptor gene locus is associated with abdominal obesity and dysregulation of the hypothalamic-pituitary-adrenal axis. Obesity Research, in press.

Shively CA, Laber-Laird K \& Auton RF (1997) Behavior and physiology of social stress and depression in female Cynomolgus monkeys. Biological Psychiatry 41, 871-882.

Smyth J, Ockenfels MC, Porter L, Kirschbaum C, Hellhammer DH \& Stone AA (1998) Stressors and mood measured on a momentary basis are associated with salivary cortisol secretion. Psychoneuroendocrinology 23, 353-370.
Vague J (1947) La différentiaton sexuelle. Facteur déterminant des formes de l'obésité. Presse Medical 55, 339-341.

Watts GCH, Harrap SB, Foy CJW, Holton DW, Edwards HV, Davidsson HR, Connor JM, Lever AF \& Fraser R (1992) Abnormalities of glucocorticoid metabolism and reninangiotensin system: a four-corners approach to the identification of genetic determinants of blood pressure. Journal of Hypertension 10, 473-482.

Weaver JU, Hitman GA \& Kopelman PG (1992) An association between a $\mathrm{Bcl}$ I restriction fragment length polymorphism of the glucocorticoid receptor locus and hyperinsulinemia in obese women. Journal of Molecular Endocrinology 9, 295-300.

WHO (1998) Obesity. Preventing and Managing the Global Epidemic. Report of WHO consultation on obesity, Geneva, 3-5 June, 1997. WHO/NUT/NCD/981. 